\title{
Stéphane CoviAUX, La fin du monde viking
}

\section{Lucie Malbos}

\section{OpenEdition \\ Journals}

Édition électronique

URL : https://journals.openedition.org/ccm/5377

DOl : $10.4000 / \mathrm{ccm} .5377$

ISSN : 2119-1026

\section{Éditeur}

Centre d'études supérieures de civilisation médiévale/Université de Poitiers

\section{Édition imprimée}

Date de publication : 1 décembre 2020

Pagination : 273-276

ISBN : 978-2-490783-07-6

ISSN : 0007-9731

\section{Référence électronique}

Lucie Malbos, "Stéphane coviaux, La fin du monde viking », Cahiers de civilisation médiévale [En ligne], 252 | 2020, mis en ligne le 02 décembre 2020, consulté le 25 novembre 2022. URL : http:// journals.openedition.org/ccm/5377 ; DOI : https://doi.org/10.4000/ccm.5377

\section{(c) (i) $\odot$}

Creative Commons - Attribution - Pas d'Utilisation Commerciale - Pas de Modification 4.0 International - CC BY-NC-ND 4.0

https://creativecommons.org/licenses/by-nc-nd/4.0/ 
Stéphane Coviaux, La fin du monde viking, Paris, Passés Composés, 2019.

Après sa thèse sur la Christianisation et naissance d'un épiscopat norvégien : l'exemple de la Norvège $d u X^{e} a u$ XII siècle, soutenue en 2003 à Paris I sous la direction de Michel Parisse, Stéphane Coviaux, professeur d'histoire en classes préparatoires littéraires, nous propose un ouvrage de synthèse sur la question complexe de la christianisation du Nord. Il est paru chez un nouvel éditeur, lancé en mars 2019 et qui compte déjà à son catalogue un certain nombre de beaux titres, dont plusieurs concernent le monde nordique. En dépit de la grande proximité thématique, cet ouvrage n'est pas la publication tardive de cette thèse : plus large, dépassant le seul cadre norvégien, il intègre très largement les évolutions historiographiques et nouvelles données, nombreuses depuis 2003, pour proposer une synthèse - en français, ce qui manquait jusqu'alors, comme le souligne l'a. dès l'introduction - parfaitement à jour de la question. Reprenant les grandes questions soulevées ces dernières années, notamment par les récentes découvertes archéologiques, mais aussi la relecture de certains textes qui en a découlé, S. Coviaux nous livre ici un ouvrage aussi clair que stimulant.

S. Coviaux annonce dès l'introduction les idées centrales de son ouvrage : le passage du paganisme nordique au christianisme ne doit pas être conçu comme une «substitution» (p. 12), mais plutôt comme une longue phase de transition, marquée par des phénomènes de persistance, d'interpénétration, avec des relectures chrétiennes de motifs et personnages païens (comme Sigurd, le tueur de dragons, qui ouvre la réflexion et est l'illustration retenue pour la couverture), un «véritable changement de civilisation », aux conséquences religieuses, mais aussi politiques, sociales et culturelles. Concevoir ainsi la christianisation du Nord pose le problème de la chronologie, délicate à fixer de façon tranchée : S. Coviaux reprend ici les trois phases proposées par le norvégien Fridtjov Birkeli, qui distinguait une longue phase d'infiltration chrétienne, une phase missionnaire, bien plus brève et centrée sur les rois, et une phase d'organisation de l'Église (Norske steinkors i tidlig middelalder: Et bidrag til belysning av overgangen fra norrøn religion til kristendom, Oslo, Universitetsforlaget [S, 10], 1973). S. Coviaux nous met toutefois en garde contre la généralisation un peu rapide de ce modèle appliqué à toute la Scandinavie : la chronologie de la conversion suédoise ne suit par exemple pas tout à fait ce schéma, qui nécessite donc d'être nuancé, adapté en fonction des régions étudiées, mais qui n'en demeure pas moins le fil 
directeur de l'ouvrage, ses apports et sa clarté l'emportant sur ses limites. Cette approche a en particulier eu le mérite de mettre en lumière la diversité des acteurs au cœur de ce processus de christianisation : missionnaires et rois certes, mais aussi marchands, esclaves, guerriers ont contribué aux multiples «négociations culturelles » ayant abouti à la conversion du Nord. En reprenant le modèle de F. Birkeli, S. Coviaux s'inscrit également dans le mouvement de «décolonisation» de l'histoire scandinave, dans la lignée des travaux de Pierre Bauduin et de ceux de Judith Jesch (The Viking Diaspora, Abingdon/ New York, Routledge [The Medieval World], 2015) notamment, qui pensent les mobilités des vikings en termes de «diaspora» (p. 15) : l'histoire de la christianisation du Nord ne saurait se réduire à celle d'une religion imposée de l'extérieur, mais doit également être pensée comme un mouvement largement initié à l'intérieur même du monde scandinave, idée aussi au cœur des travaux d'Anders Winroth (The Conversion of Scandinavia: Vikings, Merchants, and Missionaries in the Remaking of Northern Europe, New Haven/ Londres, Yale University Press, 2012).

Pour mener à bien sa réflexion, S. Coviaux s'appuie sur des sources hétérogènes, d'usage souvent délicat pour l'historien, ne reculant pas devant les problèmes et débats suscités par certaines, à commencer par les célèbres sagas, qu'il tient à pleinement intégrer pour leur dimension mémorielle et identitaire. La diversité des sources mobilisées est également rappelée par la présence, fort utile et à propos, du cahier iconographique en couleurs au centre de l'ouvrage, qui vient s'ajouter à la carte initiale et à quelques dessins insérés dans le texte : objets, pierres runiques, photographies de sites et de reconstitutions viennent appuyer - et pas simplement illustrer - la démonstration de l'a., à condition d'avoir la curiosité de s'y arrêter. On peut en effet regretter que ce cahier reste un peu marginal dans l'ouvrage et ne soit pas mieux relié au propos (par des renvois aux illustrations dans le texte, comme à la p. 265, qui évoque à la fois le crucifix de l'église de Lisbjerg et la grande pierre de Jelling, sans préciser qu'on peut en trouver des photographies au centre de l'ouvrage), ni intégré dans les index finaux (du reste fort pratiques pour circuler dans l'ouvrage, cahier central mis à part donc).

Le propos de S. Coviaux s'articule en sept chapitres, suivant une progression chronologique qui reprend en partie, comme il l'a annoncé, le schéma de F. Birkeli, mais sans respecter strictement les trois phases: en entremêlant en partie les deux premières dans les premiers chapitres, S. Coviaux montre de façon tout à fait convaincante les limites du modèle, conçu ici comme un cadre utile, mais non contraignant, dont il n'hésite pas à s'affranchir pour mieux faire ressortir la complexité du processus de christianisation. Loin d'être strictement linéaire, ce dernier doit davantage être envisagé comme une évolution sinueuse, aux nuances infinies selon les espaces.

Le chap. 1 s'ouvre sur les raids vikings et le traumatisme qu'ils constituèrent pour le monde occidental : l'altérité religieuse est, dès le tournant des $\mathrm{VIII}^{\mathrm{e}}$ et $\mathrm{IX}^{\mathrm{e}}$ s., la clé de lecture des écrits laissés par les auteurs chrétiens, à la fois terrorisés par la violence des hommes venus du Nord et inquiets de voir leur univers chrétien gagné par le péché. Mais S. Coviaux rappelle l'importance de réinscrire ces événements dans un temps plus long : les archéologues ont mis en évidence l'existence de contacts entre mondes scandinave et occidental dès les $\mathrm{IV}^{\mathrm{e}}-\mathrm{V}^{\mathrm{e}} \mathrm{s}$. Certains motifs chrétiens ont ainsi pu gagner le Nord bien avant les premiers raids. S. Coviaux ne se laisse ainsi pas enfermer dans la chronologie traditionnelle de l'époque viking (qu'on fait habituellement débuter à la fin du viII ${ }^{\mathrm{e}} \mathrm{s}$. et qu'on clôt au milieu du XI ${ }^{\mathrm{e}} \mathrm{s}$.), qui ne permet pas d'appréhender les circulations entre les deux univers religieux dans toute sa complexité et sa globalité.

Le missionnaire devenu saint, Anschaire, figure centrale dans les textes, est l'occasion de souligner, dans le chapitre suivant, l'étroite imbrication entre enjeux religieux et politiques, déjà perceptible à travers le baptême de Harald Klak. En replaçant la Vie de saint Anschaire dans son contexte de rédaction, S. Coviaux rappelle toutefois utilement le projet d'écriture qui a motivé ce texte : il s'agit avant tout, dans les années 870 , de relancer la mission dans le Nord. L'œuvre missionnaire d'Anschaire et ses suites permettent aussi de souligner que la conversion du Nord ne fut pas un processus linéaire : des phases de rejet du christianisme, comme dans les années 840 , peuvent succéder assez rapidement à des phases de tolérance.

Le chap. 3 s'ouvre sur les écrits d'Adam de Brême, mobilisés avec la prudence qui s'impose, et s'intéresse au long processus de pénétration et de diffusion des influences chrétiennes en Scandinavie, dans ses dimensions religieuse, sociale, politique et même économique. Le rôle du rituel de la prima signatio, tombé en désuétude en Occident, est rappelé : en contexte scandinave, il s'agit d'une étape intermédiaire précédant le baptême. Les premiers baptêmes apparaissant dans les textes sont ceux de rois et chefs scandinaves : ils permettent à ces derniers d'intégrer les sociétés occidentales. L'archéologie rappelle une fois encore que le changement de religion, loin d'être 
brutal, a entraîné des formes d'hybridation entre motifs chrétiens et païens (la croix et le serpent sont l'une des associations les plus fréquentes).

Comment penser la notion de conversion, conçue dans un temps bref, dans le cadre d'un processus, conçu lui sur un temps long, qui aboutit au passage de la Scandinavie au christianisme? C'est tout l'enjeu du chap. 4, dans lequel la «conversion» est entendue au sens d' «institutionnalisation du christianisme» (p. 113), à l'image de la décision prise en Islande en 999 ou 1000. Ce chapitre est l'occasion de revenir sur le rôle moteur des rois scandinaves dans l'officialisation du christianisme en Scandinavie. Les différents exemples développés (Danemark, Norvège, Islande et Suède) mettent en lumière d'importants contrastes régionaux, tout en soulignant le lien étroit entre le processus de christianisation et celui d'émergence d'un pouvoir royal fort, à l'exception de l'Islande, dépourvue de roi. S. Coviaux ne masque rien des difficultés que les sources posent pour faire cette histoire, largement dépendante des textes continentaux pour le Danemark et la Suède, de la tradition hagiographique et des sagas pour la Norvège et l'Islande, sans oublier les pierres runiques, particulièrement précieuses pour la Suède.

Les personnages opérant «dans l'ombre des rois», les missionnaires, occupent le chapitre suivant. Contrairement au monde occidental qui a vu se développer une abondante littérature hagiographique autour de ces figures, dans le Nord, les rois leur ont «volé la vedette» (p. 164). Pour cerner le rôle des acteurs ecclésiastiques, l'historien est largement dépendant des écrits d'Adam de Brême, remplis de distorsions, exagérations, passages sous silence, et ne pouvant se lire sans avoir à l'esprit le contexte de l'archevêché d'Hambourg-Brême au XI ${ }^{\mathrm{e}}$ s., comme le rappelle l'a. Ce qui ressort malgré tout de cette pauvreté documentaire, ce sont les origines assez variées des missionnaires (venus d'Angleterre, de l'Empire germanique, peut-être même du monde orthodoxe) et le fait qu'il s'agit souvent d'évêques, à la tête de diocèses dans un premier temps encore mal définis, conclusions qui se dégageaient également de la thèse de l'a. Le discours des missionnaires est une nouvelle illustration de la perméabilité des systèmes de croyances et des phénomènes d'hybridation à l'œuvre durant la période de conversion : en s'adaptant à la réalité scandinave, il a facilité la circulation des idées, établissant des passerelles entre paganisme et christianisme.

La délicate question de la perception et de la réception de la conversion par les hommes du Nord est au cœur du chap. 6 : résistance? Acceptation? Hésitation?
Résignation? Les sources textuelles et archéologiques mettent en lumière la diversité des réactions suscitées, mais sont utilisées comme il se doit : avec la plus grande prudence. Le changement de religion eut des répercussions bien au-delà de la seule religion : c'est toute l'organisation sociale qui s'en trouve bouleversée, comme en témoigne en particulier l'étude des codes de lois des $\mathrm{XI}^{\mathrm{e}}$-XIII ${ }^{\mathrm{e}} \mathrm{s}$. « $\mathrm{L}$ 'esprit de compromis et d'accommodement $»$ des autorités en a garanti le succès (p. 219), notions qui permettent par ailleurs de dépasser la présentation binaire acceptation/refus. Mais l'a. n'occulte pas pour autant les résistances ou hésitations qui ont pu exister, en revenant notamment sur le concept de «renaissance païenne» et en s'appuyant notamment sur les strophes des scaldes. S. Coviaux montre bien ici la diversité des acteurs intervenant dans l'adoption du christianisme : le rôle des élites et des femmes en particulier est mis en avant, à travers les inscriptions des pierres runiques notamment.

Le dernier chapitre est consacré à l'organisation des Églises scandinaves, qui marque la fin de la christianisation, avec la création des provinces ecclésiastiques, mais aussi plusieurs fondations monastiques et la construction de nombreuses églises, en lien avec la mise en place d'un système paroissial. Dans le même temps, la documentation écrite évolue, avec notamment le développement de la littérature homilétique au XII ${ }^{e} \mathrm{~s}$. Ce chapitre est l'occasion de proposer un bilan de l'impact majeur qu'a eu la christianisation sur les sociétés scandinaves. Devenues chrétiennes, elles s'organisent autour de nouveaux rituels, d'un nouveau calendrier, de nouveaux modèles, de nouvelles pratiques (funéraires en particulier). L'approche nuancée de $\mathrm{S}$. Coviaux permet de mettre en lumière des formes de survivance de l'ancien paganisme dans l'art nordique médiéval, tant iconographique que scaldique, faisant des scaldes les «gardiens» de la mémoire du Nord (p. 273). L'introduction de la langue et de l'alphabet latin eut également des répercussions culturelles importantes; et les représentations autour du genre et du pouvoir royal furent profondément modifiées, dans un contexte de stabilisation politique et de renforcement de l'autorité royale.

S. Coviaux nous livre ainsi une synthèse tout en nuances, qui s'appuie sur une bibliographie solide et polyglotte (intégrant largement les publications en langues scandinaves) et rend bien compte de la complexité de ce phénomène, qui a suivi une progression différente selon les régions (Scandinavie même, Angleterre, Irlande, Normandie, Islande, Féroé...) : chaque région qui fait partie de la diaspora viking suit un cheminement qui lui est propre. L'apparent 
paradoxe d'un ouvrage qui porte sur la «fin du monde viking» et s'ouvre sur ses débuts nous rappelle également les limites de toute périodisation. Cette approche vise à la fois à décloisonner les périodes habituellement définies, mais aussi les disciplines, comme c'est désormais la tendance dans les études vikings, faisant dialoguer historiens, archéologues, généticiens, historiens de l'art, épigraphistes, géographes... Face à l'ampleur du sujet, S. Coviaux a, fort judicieusement, renoncé à toute tentative d'exhaustivité (impossible sur un tel sujet), pour se concentrer sur quelques épisodes emblématiques, comme le baptême de Harald Klak ou l'œuvre missionnaire d'Anschaire. Il n'hésite pas à citer assez longuement et régulièrement les textes mobilisés, qu'il maîtrise très bien, ce qui a l'avantage de bien étayer son propos et d'intégrer les sources dans le texte (en notes, elles auraient été rejetées en fin d'ouvrage), tout en l'alourdissant parfois un peu (des pages entières étant consacrées à la citation des sources). L'ensemble de la réflexion s'organise autour de notions au cœur de l'historiographie actuelle, mettant bien en lumière la dimension progressive et négociée de ce processus de christianisation : celles de dialogue, de compromis, d'accommodement, de syncrétisme, d'hybridation, de continuité entre les deux systèmes de croyances. C'est également un écho aux travaux précédents de S. Coviaux : on retrouve notamment les figures de l'évêque missionnaire ou du saint (en particulier du saint roi), déjà au cœur de sa thèse et d'articles précédents, mais insérées ici dans une approche plus globale.

Historien des textes et traducteur d'écrits en langue norroise, S. Coviaux fait preuve d'une excellente connaissance de ce matériau. On le sent en revanche un peu moins à l'aise par moments avec les données archéologiques (funéraires en particulier), qui s'inscrivent dans des temporalités différentes et ne répondent pas toujours aux mêmes problématiques que les textes. La présence d'objets chrétiens ne suffit pas à identifier des tombes comme «chrétiennes» (p. 76-77), en raison même de la grande perméabilité des systèmes de croyances et de la longue période de transition soulignées par l'a. lui-même à certains moments. Tout en étant conscient des problèmes d'interprétation (p. 84-85), $\mathrm{S}$. Coviaux reste prisonnier de travaux un peu anciens (publiés dans les années 1980, notamment ceux d'Anne-Sofie Gräslund) et n'intègre pas vraiment les réflexions plus récentes menées sur la polysémie des données funéraires (on pense notamment, en français, aux travaux d'Alain Testart, de Luc Baray, ou encore de Chloé Belard).

La «matière du Nord» est d'un abord complexe et difficile, comme le rappelait l'a. lui-même dès les premières pages (p. 16), entraînant des choix, nécessairement subjectifs; mais c'est aussi ce qui rend toute réflexion sur le sujet particulièrement stimulante. S. Coviaux nous propose ainsi une très belle synthèse, tenant compte à la fois des travaux récents d'autres chercheurs et de ses propres recherches, comblant ainsi le vide historiographique en langue française qu'il souligne lui-même dès l'introduction. Le résultat valait bien les seize années d'attente, tout en faisant regretter, du même coup, les publications trop rares de S. Coviaux.

Lucie MaLBOS

UMR 7302 - CESCM Université de Poitiers 